\title{
Percutaneous cryoablation of osteoblastoma in the proximal femur
}

\author{
F Poullain ${ }^{1} \cdot$ JC Mattei $^{2} \cdot$ A Rochwerger $^{2} \cdot$ C Bouvier $^{3} \cdot$ C Chagnaud $^{1} \cdot$ P Champsaur $^{1,4} \cdot$ T Le Corroller $^{1,4}$
}

\begin{abstract}
A 37-year-old man presented with a 2-year history of left hip pain. Pretherapeutic imaging demonstrated a 4 $\mathrm{cm}$ osteoblastoma located in the intertrochanteric region of the proximal femur, surrounded by extensive bone marrow edema. After multidisciplinary meeting, percutaneous cryoablation was decided and performed under computed tomography guidance using three cryoprobes to match the exact size and shape of the tumor, resulting in complete resolution of symptoms. Magnetic resonance imaging follow-up demonstrated resolution of the bone marrow edema pattern and ingrowth of fat at the periphery of the ablation zone consistent with long-term healing of the tumor.
\end{abstract}

Keywords Bone $\cdot$ Femur $\cdot$ Osteoblastoma $\cdot$ Cryoablation

Osteoblastoma is a benign, bone-forming tumor that shares histological features with osteoid osteoma, but is not growth-limited, commonly being $20 \mathrm{~mm}$ or larger at diagnosis $[1,2]$. Over the past decades, surgery represented the standard treatment for osteoblastoma with either en bloc resection or curettage with grafting. Yet, the local recurrence rate following surgery was relatively high, especially after curettage and packing [3]. On the other hand, image-guided percutaneous treatments are playing an increasing role in the management of bone tumors, with higher rates of technical success, decreased morbidity, and lower costs [4]. Despite multiple advantages over heat-based ablation techniques, experience with cryoablation in the treatment of benign bone tumors is still limited [4-6]. We herein report a case of proximal femur osteoblastoma treated by percutaneous cryoablation under $\mathrm{CT}$ guidance.

Thomas.LeCorroller@ap-hm.fr

Radiology Department, APHM, Marseille, France

2 Department of Orthopedic Surgery, Hôpital Nord, APHM, Marseille, France

3 Department of Pathology, CHRU Timone, Marseille, France

4 Aix-Marseille Université, CNRS, ISM UMR 7287, Marseille, France

\section{Case report}

A 37-year-old man presented with a 2-year history of left hip pain. The discomfort was present at rest and was exacerbated by weight-bearing and walking. There was no history of previous trauma or surgery. Radiograph revealed a well-defined, lucent lesion within the intertrochanteric region of the left femur. CT showed a slightly expansile, 4-cm osteolytic tumor with partial matrix mineralization and sclerotic margins involving the lesser trochanter (Fig. 1). At magnetic resonance (MR) imaging, the lesion presented nonspecific hypointense signal on T1-weighted spin echo images and hyperintense signal on STIR and was characterized by extensive surrounding bone marrow edema (Fig. 2). Overall, the clinical and imaging features were suggestive of a benign osteogenic tumor of the proximal femur.

Given the large size of the tumor, percutaneous cryoablation with simultaneous placement of multiple probes under CT guidance was considered the safer option and was agreed upon after multidisciplinary meeting. Institutional review board (IRB) approval was not required because, for benign bone tumors, percutaneous cryoablation including its MR follow-up is considered standard of care in our institution. The procedure was performed on an inpatient basis, under general anesthesia, and strict surgical aseptic condition. A battery-powered 
Fig. 1 Coronal (a) and horizontal (b) CT reformatted images demonstrating a slightly expansile, 4-cm osteolytic tumor with partial matrix mineralization and sclerotic margins involving the lesser trochanter
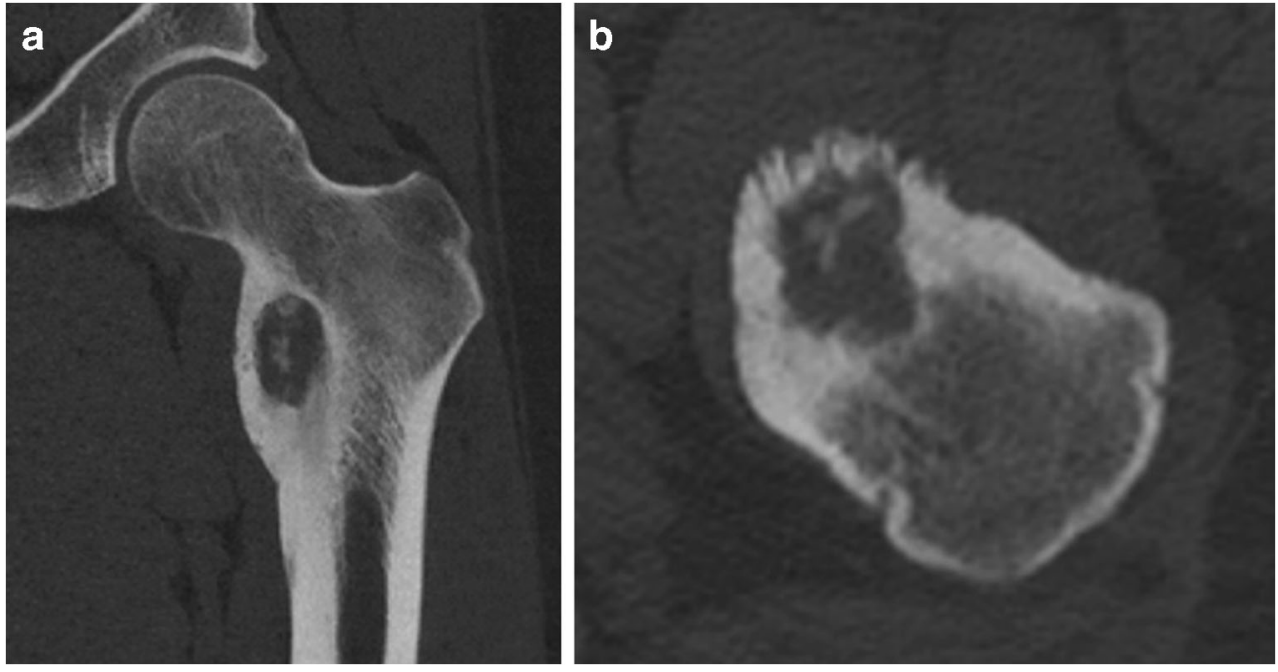

drill system (Arrow OnControl Teleflex) was used to insert three $10 \mathrm{G}$ biopsy needles into the bone up to the nidus margin with a lateral approach, which was agreed on with an oncology orthopedic surgeon to prevent complication if a different diagnosis was made at histological examination after cryoablation treatment. Bone biopsies were performed before ablation using a $12 \mathrm{G}$ trephine needle, not only to confirm diagnosis but also as part of the lesion access for the cryoprobes. Then, three cryoprobes, two Iceseeds, and one Icesphere (BTG, Boston Scientific), respectively, were placed coaxially within the nidus through the access needles. The probes were spaced $1-\mathrm{cm}$ apart (Fig. 3). The access needles were finally withdrawn slightly so that the distal $2 \mathrm{~cm}$ of the cryoprobes were exposed. A single freeze-thaw-freeze cycle was performed with a time frame consisting of two 10-min freezing phases around an intermediate 8-min thawing phase, divided into a 7 -min passive thawing and a 1 -min active thawing. Non-contrast CT scanning was performed periodically during the procedure to visualize the ablation zone and to monitor changes in surrounding tissues. The patient was observed for $60 \mathrm{~min}$ in the radiology department, then returned to the ward, and was discharged the next day.

Histological examination confirmed the diagnosis of osteoblastoma. The patient was primarily reassessed by the radiologist at 6 weeks after cryoablation. A complete pain relief was noted, without the use of any pain medication and with full return to normal activities. A follow-up MR examination was performed on the same day to evaluate the ablation zone and identify potential complications, which demonstrated complete resolution of surrounding bone marrow edema. Secondary clinical and MR follow-ups were obtained at 6 and 12 months.
There was no evidence of recurring symptoms. Interestingly, T1-weighted MR images at 1-year followup disclosed ingrowth of fat at the periphery of the ablation zone consistent with long-term healing of the tumor (Fig. 4).

\section{Discussion}

Osteoblastoma and osteoid osteoma are benign boneforming tumors, both composed of reactive-appearing woven bone with intermixed benign-appearing osteoblasts, and can be histologically indistinguishable [2, 7]. However, osteoblastoma and osteoid osteoma are considered as separate entities in the most recent WHO classification of soft tissue and bone tumors because of the risk of local recurrence for osteoblastoma [7]. Practically, the distinction between osteoblastoma and osteoid osteoma is based on clinical and radiological differences. Pain might be more progressive, but, in contrast to osteoid osteoma, discomfort may not be any worse at night; and aspirin and nonsteroidal anti-inflammatory drugs (NSAID) often fail in achieving effective pain relief. Radiologically, those lesions may be distinguished by size, with tumors larger than $15-20 \mathrm{~mm}$ classified as osteoblastomas, and the other as osteoid osteomas [7]. These size criteria could also help to predict the behavior of these lesions with smaller tumors having a lower chance of progression. Osteoblastomas often affect the spine, particularly the posterior vertebral elements. The second most common anatomical site is the metaphysis of the long bones of the extremities.

Image-guided percutaneous treatments are playing an increasing role in the management of bone tumors [4]. 

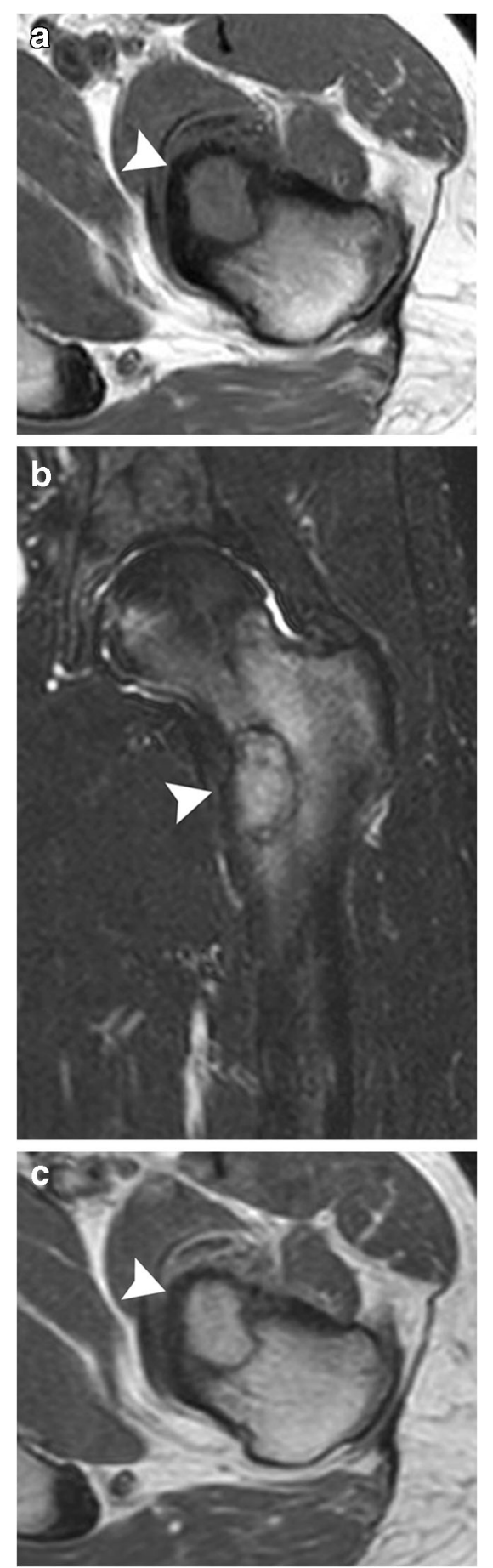

Fig. 2 At MR imaging, the lesion (arrowhead) presented nonspecific hypointense signal on T1-weighted spin echo images (a) and hyperintense signal on STIR (b) and was characterized by extensive surrounding bone marrow edema. After intravenous contrast injection (c), the tumor exhibited marked and homogenous enhancement

Because radiofrequency (RF) ablation is considered the standard treatment of osteoid osteoma, this heat-based technology was first investigated in the treatment of osteoblastoma, with reported clinical outcome similar to that achieved by surgery [8]. Yet, RF presents significant limitations in the treatment of bone-forming lesions [5]. Most importantly, osteoblastoma usually presents high tissue impedance, in particular in cortical locations, or when there is a large sclerotic rim. Because of the inductive nature of RF heat generation, RF ablation of sclerotic or heavily mineralized lesions has to be performed by using low-powered generators and by raising gradually the temperature to obtain complete destruction of tissue in the ablation zone, which significantly increases the ablation time. In addition, the treatment of large bone tumors requires overlapping RF ablations which increases the procedure duration and may leave residual tumor at the ablation interfaces [4].

On the other hand, cryoablation has recently been described in the treatment of osteoid osteoma affording distinct advantages over heat-based ablation techniques [9, 10]. First, in contrast to RF ablation, the ablation zone generated with cryoablation can be readily monitored during the procedure with intermittent non-contrast CT. A second advantage is the opportunity to use multiple cryoprobes simultaneously with a synergistic effect, allowing for rapid generation of a large volume of ice with the ability to tailor the iceball to the tumor size and morphology. Third, as opposed to the inductive nature of RF heat generation, the conductive nature of ice formation allows the iceball transmission across sclerotic bone, which is particularly useful when treating osteoblastoma. Lastly, cryoablation offers intrinsic anesthetic properties providing reduction of immediate postprocedural pain compared with RF ablation [6].

In 2015, percutaneous cryoablation of a large pelvic osteoblastoma recurring after surgery was reported for the first time [11]. The authors observed excellent clinical results with durable pain relief and absence of recurrence at long-term follow-up. In 2019, Cazzato et al. published an observational study on ten patients suggesting that cryoablation represents an effective therapeutic option for painful osteoblastomas [12]. In comparison with the present case, the osteoblastomas treated in their series were significantly smaller, with a size ranging between 16 and $28 \mathrm{~mm}$, and most often located in the spine.

Cryoablation of bone tumor performed with curative intent is considered safe with an overall complication rate of $9.1 \%$ and a major complication rate of $2.5 \%$ $[13,14]$. The most common major complication of bone tumor cryoablation is secondary fracture, occurring in approximately $1 \%$ of patients [13]. Osteoblastomas are often located in high-risk areas such as the neural arch at the spine or metaphyseal region of long bones. In the present case, despite involvement of the most loaded part 
Fig. 3 Coronal CT reformatted image (a) exhibiting three cryoprobes placed within the nidus and spaced $1-\mathrm{cm}$ apart to obtain a synergistic effect. Sagittal CT reformatted image (b) displaying the iceball (arrowhead)
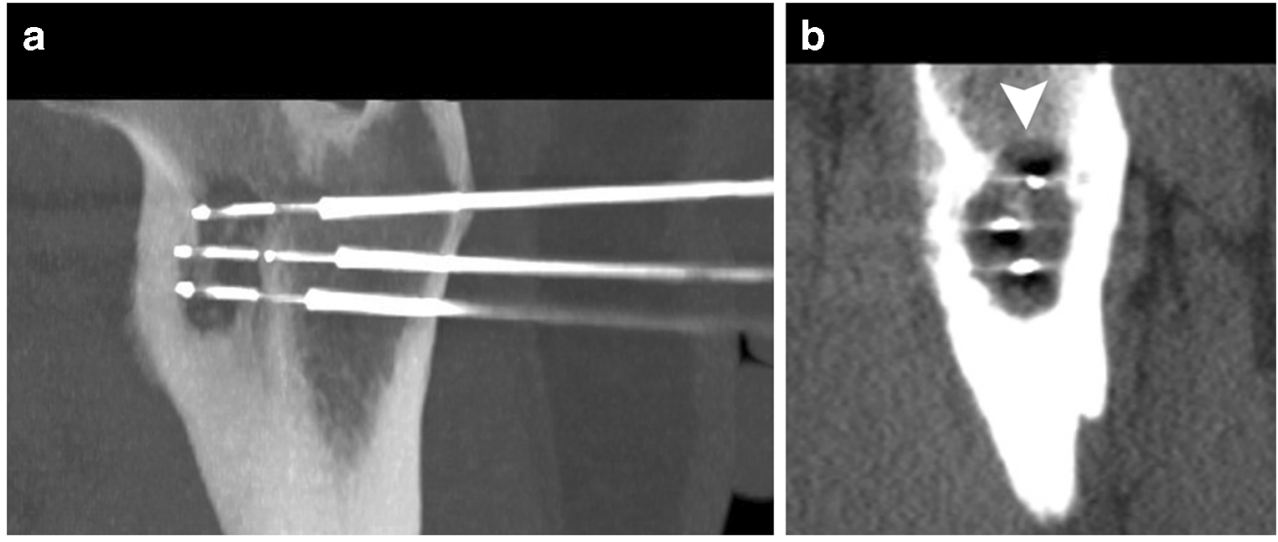

Fig. 4 Coronal T1-weighted follow-up MR image at 6 weeks (a) and 1 year (b) after ablation disclosing ingrowth of fat at the periphery of the ablation zone consistent with long-term healing of the tumor
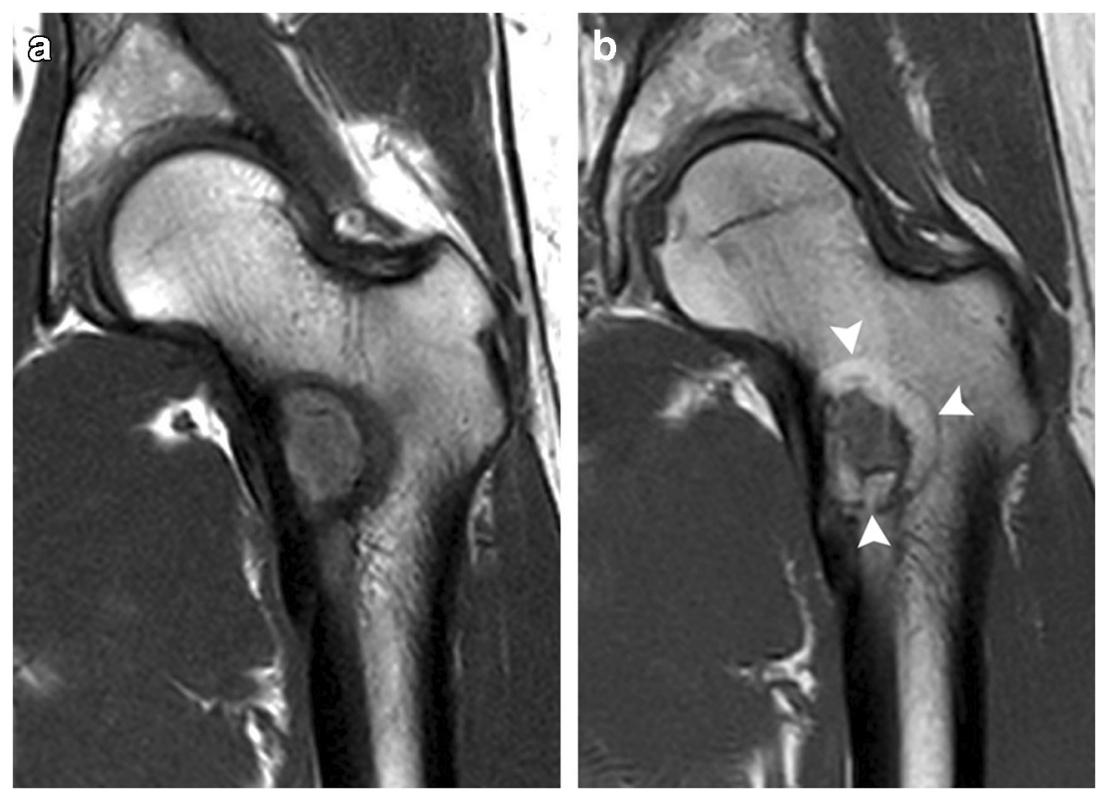

of the proximal femur, the fracture risk was manageable because the tumor exhibited thick sclerotic margins, and the cortical alteration involved less than one third of the total femur circumference.

This report suggests that percutaneous CT-guided cryoablation represents an effective therapeutic option for patients presenting with painful osteoblastoma involving the proximal femur metaphysis. Further studies including larger cohorts of patients with longer follow-ups will be needed to confirm the safety and durability of such treatment.

\section{Compliance with ethical standards}

Conflict of interest The authors declare that they have no conflict of interest.

\section{References}

1. Yalcinkaya U, Doganavsargil B, Sezak M, Kececi B, Argin M, Basdemir G, et al. Clinical and morphological characteristics of osteoid osteoma and osteoblastoma: a retrospective single-center analysis of 204 patients. Ann Diagn Pathol. 2014;18:319-25.

2. Zhang Y, Rosenberg AE. Bone-forming tumors. Surgical Pathology Clinics. 2017;10:513-35.

3. Berry M, Mankin H, Gebhardt M, Rosenberg A, Hornicek F. Osteoblastoma: a 30-year study of 99 cases. J Surg Oncol. 2008;98:179-83.

4. Rosenthal D, Callstrom MR. Critical review and state of the art in interventional oncology: benign and metastatic disease involving bone. Radiology. 2012;262:765-80.

5. Callstrom MR, Kurup AN. Percutaneous ablation for bone and soft tissue metastases-why cryoablation? Skelet Radiol. 2009;38:835-9.

6. Thacker PG, Callstrom MR, Curry TB, et al. Palliation of painful metastatic disease involving bone with imaging-guided treatment: 
Comparison of patients' immediate response to radiofrequency ablation and cryoablation. Am J Roentgenol. 2011;197:510-5.

7. Fletcher CDM, Bidge JA, Hogendoom P, et al. World Health Organisation classification of tumours, pathology and genetics. In: Tumours of soft tissue and bone, vol. 5. 4th ed: ARC; 2013. p. 277-80.

8. Weber MA, Sprengel SD, Omlor GW, et al. Clinical long-term outcome, technical success, and cost analysis of radiofrequency ablation for the treatment of osteoblastomas and spinal osteoid osteomas in comparison to open surgical resection. Skelet Radiol. 2015;44:981-93.

9. Coupal TM, Mallinson PI, Munk PL, Liu D, Clarkson P, Ouellette H. CT-guided percutaneous cryoablation for osteoid osteoma: initial experience in adults. Am J Roentgenol. 2014;202:1136-9.

10. Santiago E, Pauly V, Brun G, Guenoun D, Champsaur P, Le Corroller T. Percutaneous cryoablation for the treatment of osteoid osteoma in the adult population. Eur Radiol. 2018;28:2336-44.
11. Kumasaka S, Miyazaki M, Tsushima Y. CT- guided percutaneous cryoablation of an aggressive osteoblastoma: a case report. J Vasc Interv Radiol. 2015;26:1746-8.

12. Cazzato RL, Auloge P, Dalili D, De Marini P, Di Marco A, Garnon $\mathrm{J}$, et al. Percutaneous image-guided cryoablation of osteoblastoma. AJR Am J Roentgenol. 2019;213:1157-62.

13. Auloge P, Cazzato RL, Rousseau C, Caudrelier J, Koch G, Rao P, et al. Complications of percutaneous bone tumor cryoablation: a 10year experience. Radiology. 2019;291:521-8.

14. Jennings JW. Is percutaneous bone cryoablation safe? Radiology. 2019;291:529-30. 